Aobing Li · YanYan Li

\title{
A fully nonlinear version of the Yamabe problem on manifolds with boundary
}

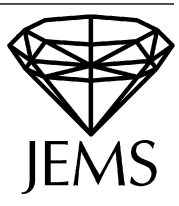

Dedicated to Antonio Ambrosetti on his 60th birthday

Received September 7, 2005

\begin{abstract}
We propose to study a fully nonlinear version of the Yamabe problem on manifolds with boundary. The boundary condition for the conformal metric is the mean curvature. We establish some Liouville type theorems and Harnack type inequalities.
\end{abstract}

\section{Introduction}

Let $(M, g)$ be an $n$-dimensional compact smooth Riemannian manifold without boundary, $n \geq 3$. The well known Yamabe conjecture states that there exist metrics which are pointwise conformal to $g$ and have constant scalar curvature. The Yamabe conjecture is proved through the work of Yamabe (1960), Trudinger (1968), Aubin (1976) and Schoen (1984). There has been much activity on fully nonlinear versions of the Yamabe problem (see, e.g., [14] and the references therein). A very general fully nonlinear version of the Yamabe problem was proposed, and solved when manifolds are locally conformally flat, in joint work with Aobing Li (see [12] and [14], and in particular Conjecture 1.1-1.1' and Theorem 1.1-1.1' in [14]). In the present paper, we start to look at analogues on manifolds with boundary.

If we let $\left(M^{n}, g\right)$ denote some smooth compact $n$-dimensional Riemannian manifold with boundary, an analogous problem is to find conformal metrics with constant scalar curvature and constant boundary mean curvature. The problem has been studied by many authors: see, e.g., Cherrier ([5])-[9]), Han and Li ([10] and [11]), Ambrosetti, Malchiodi and $\mathrm{Li}([1])$, Brendle ([3]), and the references therein. This boundary Yamabe problem is called of positive type if the first eigenvalue of

$$
\begin{cases}-L_{g} \varphi=\lambda \varphi & \text { in } M^{\circ}, \\ \frac{\partial \varphi}{\partial \nu}+\frac{n-2}{2} h_{g} \varphi=0 & \text { on } \partial M,\end{cases}
$$

is positive, where $h_{g}$ denotes the mean curvature.

A. Li: Department of Mathematics, Rutgers University, 110 Frelinghuysen Rd., Piscataway, NJ 08854, USA; current address: Department of Mathematics, University of Wisconsin, 480 Lincoln Drive, Madison, WI 53706, USA; e-mail: aobingli@math.wisc.edu Y.Y. Li: Department of Mathematics, Rutgers University, 110 Frelinghuysen Rd., Piscataway, NJ 08854, USA; e-mail: yyli@ math.rutgers.edu 
We consider an extension of the boundary Yamabe problem of positive type to the following fully nonlinear setting. Let

$$
A_{g}:=\frac{1}{n-2}\left(\operatorname{Ric}_{g}-\frac{R_{g}}{2(n-1)} g\right)
$$

denote the Schouten tensor of $g$, where $\operatorname{Ric}_{g}$ and $R_{g}$ denote respectively the Ricci tensor and the scalar curvature of $g$. Let, as in [14], $V$ be an open convex subset of $\mathbb{R}^{n}$ which is symmetric with respect to the coordinates and has non-empty $C^{\infty}$ boundary $\partial V$. For $\lambda \in \partial V$, let $v(\lambda)$ denote the inner unit normal to $\partial V$. We further assume that

$$
\nu(\lambda) \in \Gamma_{n}:=\left\{\lambda \in \mathbb{R}^{n} \mid \lambda_{i}>0, \forall 1 \leq i \leq n\right\}, \quad \forall \lambda \in \partial V,
$$

and

$$
\nu(\lambda) \cdot \lambda>0, \quad \forall \lambda \in \partial V
$$

Let

$$
\Gamma(V):=\{s \lambda \mid \lambda \in V, 0<s<\infty\}
$$

be the (open convex) cone with vertex at the origin generated by $V$.

Question 1. Assume that $V$ is an open symmetric convex subset of $\mathbb{R}^{n}$, with $\emptyset \neq \partial V \in$ $C^{\infty}$ satisfying (1) and (2). Let $\left(M^{n}, g\right)$ be a compact smooth Riemannian manifold with boundary satisfying

$$
\lambda\left(A_{g}\right) \in \Gamma(V) \quad \text { on } \bar{M},
$$

and let $c \in \mathbb{R}$ be any constant. Does there exist a smooth positive function $u \in C^{\infty}(\bar{M})$ such that the conformal metric $\hat{g}=u^{4 /(n-2)} g$ satisfies

$$
\lambda\left(A_{\hat{g}}\right) \in \partial V \quad \text { on } M^{n},
$$

and the boundary mean curvature $h_{\hat{g}}$ satisfies

$$
h_{\hat{g}}=c \quad \text { on } \partial M ?
$$

For $\hat{g}=u^{4 /(n-2)} g$,

$$
A_{\hat{g}}=-\frac{2}{n-2} u^{-1} \nabla^{2} u+\frac{2 n}{(n-2)^{2}} u^{-2} \nabla u \otimes \nabla u-\frac{2}{(n-2)^{2}} u^{-2}|\nabla u|^{2} g+A_{g},
$$

where covariant derivatives on the right side are with respect to $g$. Let $g_{1}=u^{4 /(n-2)} g_{\text {flat }}$, where $g_{\text {flat }}$ denotes the Euclidean metric on $\mathbb{R}^{n}$. Then, by the above transformation formula,

$$
A_{g_{1}}=u^{4 /(n-2)} A_{i j}^{u} d x^{i} d x^{j},
$$

where

$$
\begin{aligned}
A^{u}:= & -\frac{2}{n-2} u^{-(n+2) /(n-2)} \nabla^{2} u+\frac{2 n}{(n-2)^{2}} u^{-2 n /(n-2)} \nabla u \otimes \nabla u \\
& -\frac{2}{(n-2)^{2}} u^{-2 n /(n-2)}|\nabla u|^{2} I,
\end{aligned}
$$

and $I$ is the $n \times n$ identity matrix. In this case, $\lambda\left(A_{g_{1}}\right)=\lambda\left(A^{u}\right)$ where $\lambda\left(A^{u}\right)$ denotes the eigenvalues of the $n \times n$ symmetric matrix $A^{u}$. 
Let $\psi$ be a Möbius transformation in $\mathbb{R}^{n}$, i.e., a transformation generated by translation, multiplication by nonzero constants, and the inversion $x \mapsto x /|x|^{2}$. For any positive $C^{2}$ function $u$, let $u_{\psi}:=\left|J_{\psi}\right|^{(n-2) / 2 n}(u \circ \psi)$ where $J_{\psi}$ denotes the Jacobian of $\psi$. A calculation shows that $A^{u_{\psi}}$ and $A^{u} \circ \psi$ differ only by an orthogonal conjugation and therefore

$$
\lambda\left(A^{u \psi}\right)=\lambda\left(A^{u}\right) \circ \psi .
$$

Let $\mathcal{S}^{n \times n}$ denote the set of $n \times n$ real symmetric matrices, $\mathcal{S}_{+}^{n \times n} \subset \mathcal{S}^{n \times n}$ the set of positive definite matrices, $O(n)$ the set of $n \times n$ real orthogonal matrices, $U \subset \mathcal{S}^{n \times n}$ an open set satisfying

$$
\begin{gathered}
O^{-1} U O=U, \quad \forall O \in O(n), \\
U \cap\{M+t N \mid 0<t<\infty\} \text { is convex, } \forall M \in \mathcal{S}^{n \times n}, N \in \mathcal{S}_{+}^{n \times n},
\end{gathered}
$$

and let $F \in C^{1}(U)$ satisfy

$$
\begin{gathered}
F\left(O^{-1} M O\right)=F(M), \quad \forall M \in U, O \in O(n), \\
\left(F_{i j}(M)\right)>0, \quad \forall M \in U,
\end{gathered}
$$

where $F_{i j}(M):=\frac{\partial F}{\partial M_{i j}}(M)$.

To answer Question 1, it is important to investigate the corresponding Liouville type problem on Euclidean half space. Theorems 1 and 2 below provide such Liouville type theorems.

We use $B_{R}(x)$ to denote the ball in $\mathbb{R}^{n}$ of radius $R$ and centered at $x$, and write $B_{R}=B_{R}(0)$. Let $\mathbb{R}_{+}^{n}=\left\{\left(x_{1}, \ldots, x_{n}\right) \in \mathbb{R}^{n} \mid x_{n}>0\right\}$ and $B_{1}^{+}=B_{1} \cap \mathbb{R}_{+}^{n}$. Consider, for some $c \in \mathbb{R}$,

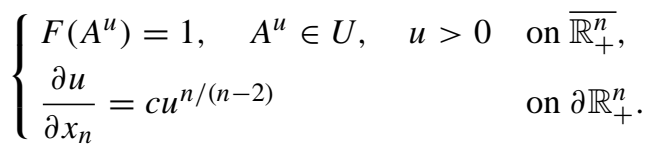

Our first result is under the assumption that the solution has good behavior near infinity.

Theorem 1. For $n \geq 3$, let $U \subset \mathcal{S}^{n \times n}$ be an open set satisfying (4) and (5), and let $F \in C^{1}(U)$ satisfy 6 and $\sqrt{77}$. For $c \in \mathbb{R}$, assume that $u \in C^{2}\left(\overline{\mathbb{R}_{+}^{n}}\right)$ is a solution of 8 , satisfying, for $u_{0,1}(x):=|x|^{2-n} u\left(x /|x|^{2}\right)$,

$u_{0,1}$ can be extended to a positive continuous function in $\overline{B_{1}^{+}}$,

$$
\limsup _{x \rightarrow 0} x \cdot \nabla u_{0,1}(x)<\frac{n-2}{2} u_{0,1}(0),
$$

and

$$
\lim _{x \rightarrow 0}|x|^{2} \nabla u_{0,1}(x)=0
$$

Then

$$
u\left(x^{\prime}, x_{n}\right) \equiv\left(\frac{a}{1+b\left|\left(x^{\prime}, x_{n}\right)-\left(\bar{x}^{\prime}, \bar{x}_{n}\right)\right|^{2}}\right)^{(n-2) / 2} \quad \text { on } \mathbb{R}_{+}^{n}
$$


where $\bar{x}=\left(\bar{x}^{\prime}, \bar{x}_{n}\right) \in \mathbb{R}^{n}, a>0$ and $b+\left(\min \bar{x}_{n}, 0\right)^{2}>0$ are two constants satisfying $2 a^{-2} b I \in U, F\left(2 a^{-2} b I\right)=1$ and $(n-2) a^{-1} b \bar{x}_{n}=c$.

Remark 1. In the above theorem, we do not assume $u$ to be superharmonic.

Corollary 1. For $n \geq 3$, let $U \subset \mathcal{S}^{n \times n}$ be an open set satisfying (4) and (5), and let $F \in C^{1}(U)$ satisfy $(6)$ and $\left(77\right.$. Assume that $u \in C^{2}\left(\overline{B_{1}}\right)$ satisfies, for some $c \in \mathbb{R}$,

$$
\begin{cases}F\left(A^{u}\right)=1, \quad A^{u} \in U, \quad u>0 & \text { in } \overline{B_{1}}, \\ \frac{\partial u}{\partial v}+\frac{n-2}{2} u=-c u^{n /(n-2)} & \text { on } \partial B_{1},\end{cases}
$$

where $v$ denotes the unit outer normal to $\partial B_{1}$. Then $u$ is of the form

$$
u(x) \equiv\left(\frac{a}{1+b|x-\bar{x}|^{2}}\right)^{(n-2) / 2} \quad \text { in } B_{1},
$$

where $a, b \in \mathbb{R}$ and $\bar{x} \in \mathbb{R}^{n}$ satisfy

$$
a>0, \quad \frac{2 b}{a^{2}} I \in U, \quad F\left(\frac{2 b}{a^{2}} I\right)=1, \quad \frac{n-2}{2}\left(1+b|\bar{x}|^{2}-b\right)=-c a .
$$

Our next Liouville type theorem does not require any hypothesis on the solution near infinity.

Theorem 2. For $n \geq 3$, let $U \subset \mathcal{S}^{n \times n}$ be an open set satisfying (4) and (5), and let $F \in C^{1}(U)$ satisfy (6) and (7). Assume that

$$
0 \notin \overline{F^{-1}(1)} \text {. }
$$

For $c \in \mathbb{R}$, assume that $u \in C^{2}\left(\overline{\mathbb{R}_{+}^{n}}\right)$ is a solution of $(8)$ satisfying

$$
\Delta u \leq 0 \text { in } \mathbb{R}_{+}^{n} .
$$

Then $u$ is of the form (10) with $\bar{x}$, $a$ and $b$ given below 100 .

Remark 2. For $c \leq 0$, the assumption (11) is not needed. This can be seen in the proof.

For $M \in \mathcal{S}^{n \times n}$, let $\left(\lambda_{1}(M), \ldots, \lambda_{n}(M)\right)$ denote its eigenvalues. Set

$$
\begin{aligned}
F_{k}(M) & :=\sum_{1 \leq i_{1}<\cdots<i_{k} \leq n} \lambda_{i_{1}}(M) \cdots \lambda_{i_{k}}(M), \\
U_{k} & :=\left\{M \in \mathcal{S}^{n \times n} \mid F_{i}(M)>0 \forall 1 \leq i \leq k\right\} .
\end{aligned}
$$

Remark 3. $(F, U)=\left(F_{k}^{1 / k}, U_{k}\right), 1 \leq k \leq n$, satisfy the hypotheses of the theorem.

Remark 4. For $(F, U)=\left(F_{1}, U_{1}\right)$, the result was proved by Li and Zhu [17]; while under an additional hypothesis $u(x)=O\left(|x|^{2-n}\right)$ for large $|x|$, the solutions were classified by Escobar [6]. 
Our proofs of Theorems 1 and 2 make use of the following result concerning radially symmetric solutions.

Theorem 3. For $n \geq 3$, let $U \subset \mathcal{S}^{n \times n}$ be an open set satisfying (4), and let $F \in C^{1}(U)$ satisfy (6) and 7). Assume that $u \in C^{2}\left(B_{1}\right)$ is radially symmetric and satisfies

$$
F\left(A^{u}\right)=1, \quad A^{u} \in U, \quad u>0 \quad \text { in } B_{1} .
$$

Then

$$
u(x) \equiv\left(\frac{a}{1+b|x|^{2}}\right)^{(n-2) / 2} \text { in } B_{1},
$$

where $a>0, b \geq-1,\left(2 b / a^{2}\right) I \in U$ and $F\left(\left(2 b / a^{2}\right) I\right)=1$.

In the following we state some of the results in a forthcoming paper [15]. First, an existence and compactness result on subcritical equations:

Theorem 4. Let $(M, g)$ be a smooth, compact, connected Riemannian manifold of dimension $n \geq 3$, and let

$$
1<1+\epsilon \leq p \leq \frac{n+2}{n-2}-\epsilon<\frac{n+2}{n-2} .
$$

Then there exists a positive solution $u \in C^{\infty}(M)$ to

$$
\sigma_{k}^{1 / k}\left(A_{u^{4 /(n-2)} g}\right)=u^{p-(n+2) /(n-2)} \quad \text { on } M .
$$

Moreover all positive solutions of (13) satisfy, for all $m \geq 2$,

$$
\|u\|_{C^{m}(M, g)}+\|1 / u\|_{C^{m}(M, g)} \leq C,
$$

where $C>0$ depends only on $\left(M^{n}, g\right), \epsilon$ and $m$.

Remark 5. For $k=1$, this is well known.

Next, a Harnack type inequality on Euclidean half balls:

Theorem 5. For $n \geq 3$ and $R>0$, let $u \in C^{2}\left(\overline{B_{3 R}^{+}}\right)$be a solution of the equation

$$
\begin{cases}\sigma_{k}^{1 / k}\left(A^{u}\right)=1 & \text { in } B_{3 R}^{+}:=B_{3 R} \cap \mathbb{R}_{+}^{n}, \\ \frac{\partial u}{\partial x_{n}}=c u^{n /(n-2)} & \text { on } \partial B_{3 R}^{+} \cap \partial \mathbb{R}_{+}^{n} \text { for some constant } c \\ u>0, A^{u} \in \Gamma_{k} & \text { on } \overline{B_{3 R}^{+}} .\end{cases}
$$

Then there exists some constant $C>0$ depending only on $n$ and $c$ such that

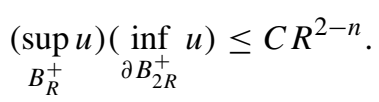

Remark 6. For $k=1$, this, as well as a stronger form, is established by Li and Zhang in [16] (see Theorem 1.7 and Remark 1.11 there).

Remark 7. Theorems 4 and 5 hold for more general $(f, \Gamma)$ (see [15]).

Theorems 1 and 2, which are Liouville type theorems on Euclidean half spaces, are extensions of Theorem 1.4 in [12] and Theorem 1.3 in [14] respectively. The present paper is essentially the second part of [13]. The first part of [13] is essentially [14]. 


\section{Proof of Theorems $1+3$}

\subsection{Proof of Theorem 3}

Let $u$ be as in Theorem 3 , and let

$$
v(r)=u(r, 0, \ldots, 0), \quad 0 \leq r<1 .
$$

Clearly, $v^{\prime}(0)=0$. For $x=(r, 0, \ldots, 0), 0<r<1$, we have

$$
\nabla u(x)=\left(v^{\prime}(r), 0, \ldots, 0\right), \quad \nabla^{2} u(x)=\operatorname{diag}\left(v^{\prime \prime}(r), \frac{v^{\prime}(r)}{r}, \ldots, \frac{v^{\prime}(r)}{r}\right),
$$

and

$$
A^{u}(x)=\operatorname{diag}\left(\lambda_{1}^{v}(r), \lambda_{2}^{v}(r), \ldots, \lambda_{n}^{v}(r)\right),
$$

where

$$
\left\{\begin{array}{l}
\lambda_{1}^{v}(r)=-\frac{2}{n-2} v^{-(n+2) /(n-2)} v^{\prime \prime}+\frac{2(n-1)}{(n-2)^{2}} v^{-2 n /(n-2)}\left(v^{\prime}\right)^{2} \\
\lambda_{2}^{v}(r)=\cdots=\lambda_{n}^{v}(r)=-\frac{2}{n-2} v^{-(n+2) /(n-2)} \frac{v^{\prime}}{r}-\frac{2}{(n-2)^{2}} v^{-2 n /(n-2)}\left(v^{\prime}\right)^{2} .
\end{array}\right.
$$

Here and in the following, we use $\operatorname{diag}\left(\lambda_{1}, \ldots, \lambda_{n}\right)$ to denote the diagonal matrix

$$
\left(\begin{array}{lll}
\lambda_{1} & & \\
& \ddots & \\
& & \lambda_{n}
\end{array}\right)
$$

Let

$$
w(x)=\left(\frac{a}{1+b|x|^{2}}\right)^{(n-2) / 2} \quad \text { with } \quad a=v(0)^{2 /(n-2)}, \quad b=\frac{1}{2-n} a^{(2-n) / 2} v^{\prime \prime}(0) .
$$

With these choices of $a$ and $b$, we have

$$
w(0)=v(0), \quad w^{\prime}(0)=v^{\prime}(0)=0, \quad w^{\prime \prime}(0)=v^{\prime \prime}(0) .
$$

A calculation yields

and therefore $w$ satisfies

$$
A^{w}(x) \equiv \frac{2 b}{a^{2}} I=A^{u}(0),
$$

$$
F\left(A^{w}\right)=1, \quad A^{w} \in U, \quad w>0 \quad \text { in }\left\{\left.x \in \mathbb{R}^{n}|b| x\right|^{2}>-1\right\} .
$$

Introduce $f\left(\lambda_{1}, \ldots, \lambda_{n}\right)=F\left(\operatorname{diag}\left(\lambda_{1}, \ldots, \lambda_{n}\right)\right)$. Clearly,

$$
\lambda_{j}(0):=\lim _{r \rightarrow 0} \lambda_{j}(r)=-\frac{2}{n-2} v(0)^{-(n+2) /(n-2)} v^{\prime \prime}(0), \quad 1 \leq j \leq n,
$$

and therefore, by the symmetry of $f$ in $\lambda_{1}, \ldots, \lambda_{n}$, we have

$$
f_{\lambda_{j}}\left(\lambda_{1}(0), \ldots, \lambda_{n}(0)\right)=f_{\lambda_{1}}\left(\lambda_{1}(0), \ldots, \lambda_{n}(0)\right), \quad 2 \leq j \leq n .
$$

Since $\operatorname{diag}\left(\lambda_{1}(0), \ldots, \lambda_{n}(0)\right) \in U$, we have, by $[7], f_{\lambda_{1}}\left(\lambda_{1}(0), \ldots, \lambda_{n}(0)\right)>0$. 
Lemma 1. Let $\alpha$ and $\beta$ be positive constants, and let $k \geq 1$ be an integer satisfying $k+\gamma>\alpha$ for some $0<\gamma \leq 1$. Assume that $\xi \in C^{k-1, \gamma}([0, \beta])$ satisfies

$$
|\xi(r)| \leq \frac{\alpha}{r} \int_{0}^{r}|\xi(s)| d s, \quad \forall 0<r<\beta,
$$

and

$$
\xi(0)=\xi^{\prime}(0)=\cdots=\xi^{(k-1)}(0)=0
$$

Then

$$
\xi \equiv 0 \quad \text { on }[0, \beta]
$$

Proof. We deduce from (15) that

$$
|\xi(r)| \leq C r^{k-1+\gamma}, \quad 0 \leq r \leq \beta,
$$

where $C$ is some positive constant. Using (17), we deduce, from (14) that

$$
|\xi(r)| \leq \frac{\alpha}{r} \int_{0}^{r} C s^{k-1+\gamma} d s=\frac{C \alpha}{k+\gamma} r^{k-1+\gamma}, \quad 0 \leq r \leq \beta .
$$

Using (18), we deduce from (14) that

$$
|\xi(r)| \leq \frac{\alpha}{r} \int_{0}^{r} \frac{C \alpha}{k+\gamma} s^{k-1+\gamma} d s=C\left(\frac{\alpha}{k+\gamma}\right)^{2} r^{k-1+\gamma}, \quad 0 \leq r \leq \beta .
$$

Continuing this way (by induction), we have

$$
|\xi(r)| \leq C\left(\frac{\alpha}{k+\gamma}\right)^{j} r^{k-1+\gamma}, \quad \forall 0 \leq r \leq \beta, \forall j=1,2, \ldots
$$

Since $\alpha /(k+\gamma)<1$, we obtain 16 by sending $j \rightarrow \infty$. Lemma 1 is established.

Continue the proof of Theorem 3 . Since

$$
1=f\left(\lambda_{1}^{v}(r), \ldots, \lambda_{n}^{v}(r)\right)=f\left(\lambda_{1}^{w}(r), \ldots, \lambda_{n}^{w}(r)\right),
$$

we have

$$
\begin{aligned}
0 & =\int_{0}^{1}\left(\frac{d}{d t} f\left(t \lambda^{v}(r)+(1-t) \lambda^{w}(r)\right)\right) d t \\
& =\left(\sum_{i=1}^{n} \int_{0}^{1} f_{\lambda_{i}}\left(t \lambda^{v}(r)+(1-t) \lambda^{w}(r)\right) d t\right)\left(\lambda_{i}^{v}(r)-\lambda_{i}^{w}(r)\right) .
\end{aligned}
$$

Since $\lambda^{v}(0)=\lambda^{w}(0)$ and $f_{\lambda_{i}}\left(\lambda^{v}(0)\right)=f_{\lambda_{1}}\left(\lambda^{v}(0)\right)>0$, we deduce from the above that

$$
\lambda_{1}^{v}(r)-\lambda_{1}^{w}(r)=-\sum_{i=2}^{n}(1+o(1))\left(\lambda_{i}^{v}(r)-\lambda_{i}^{w}(r)\right)
$$

where $o(1)$ denotes some quantities tending to 0 as $r \rightarrow 0$. 
Since $v^{\prime}(0)=w^{\prime}(0)=0$, we have

$$
\begin{aligned}
\lambda_{1}^{v}(r)-\lambda_{1}^{w}(r)= & -\frac{2}{n-2} v(r)^{-(n+2) /(n-2)}\left(v^{\prime \prime}(r)-w^{\prime \prime}(r)\right) \\
& +O(1)\left(|v(r)-w(r)|+\left|v^{\prime}(r)-w^{\prime}(r)\right|\right),
\end{aligned}
$$

and, for $2 \leq i \leq n$,

$$
\begin{aligned}
\lambda_{i}^{v}(r)-\lambda_{i}^{w}(r)= & -\frac{2}{n-2} v(r)^{-(n+2) /(n-2)} \frac{v^{\prime}(r)-w^{\prime}(r)}{r} \\
& +O(1)\left(|v(r)-w(r)|+\left|v^{\prime}(r)-w^{\prime}(r)\right|\right) .
\end{aligned}
$$

It follows that

$v^{\prime \prime}(r)-w^{\prime \prime}(r)=-\frac{n-1}{r}\left(v^{\prime}(r)-w^{\prime}(r)\right)(1+o(1))+O(1)\left(|v(r)-w(r)|+\left|v^{\prime}(r)-w^{\prime}(r)\right|\right)$, i.e.,

$\left(r^{n-1}\left(v^{\prime}(r)-w^{\prime}(r)\right)\right)^{\prime}=o\left(r^{n-2}\right)\left|v^{\prime}(r)-w^{\prime}(r)\right|+O\left(r^{n-1}\right)\left(|v(r)-w(r)|+\left|v^{\prime}(r)-w^{\prime}(r)\right|\right)$.

Integrating the above, we have, using $v(0)-w(0)=0$,

$$
\begin{aligned}
\mid v^{\prime}(r) & -w^{\prime}(r) \mid \\
& \leq \frac{o(1)}{r} \int_{0}^{r}\left|v^{\prime}(s)-w^{\prime}(s)\right| d s+C \int_{0}^{r}\left(|v(s)-w(s)|+\left|v^{\prime}(s)-w^{\prime}(s)\right|\right) d s \\
& \leq \frac{o(1)}{r} \int_{0}^{r}\left|v^{\prime}(s)-w^{\prime}(s)\right| d s .
\end{aligned}
$$

Applying Lemma 1 to $\xi=v^{\prime}-w^{\prime}$, we have, for some $\delta>0$,

$$
v^{\prime}(r)-w^{\prime}(r) \equiv 0 \quad \text { in }(0, \delta) .
$$

For $r \geq \delta$, the O.D.E. satisfied by $v$ and $w$ is regular, so $v \equiv w$ in $(0,1)$. Hence $w$ is regular in $(0,1)$. Consequently, $b \geq-1$.

\subsection{Proof of Theorem 1}

To give the main idea of the proof, we first prove Theorem 1 under a stronger assumption on $u$, i.e.,

$$
u_{0,1}(x):=|x|^{2-n} u\left(\frac{x}{|x|^{2}}\right) \text { can be extended to a positive function in } C^{2}\left(\overline{B_{1}^{+}}\right),
$$

and

$$
A^{u_{0,1}} \in U \text { on } \overline{B_{1}^{+}} \text {. }
$$

For $x \in \mathbb{R}^{n}, \lambda>0$, let $u_{x, \lambda}$ denote the reflection of $u$ with respect to $B_{\lambda}(x)$, i.e.,

$$
u_{x, \lambda}(y):=\left(\frac{\lambda}{|y-x|}\right)^{n-2} u\left(x+\frac{\lambda^{2}(y-x)}{|y-x|^{2}}\right) .
$$


Lemma 2. Let $u$ be as in Theorem 1. Then, for any $x \in \partial \mathbb{R}_{+}^{n}$, there exists $\lambda_{0}(x)>0$ such that

$$
u_{x, \lambda} \leq u \quad \text { on } \overline{\mathbb{R}_{+}^{n}} \backslash B_{\lambda}(x), \quad \forall 0<\lambda<\lambda_{0}(x) .
$$

Proof. We follow the arguments in the proof of Lemma 2.1 in [16]. Without loss of generality, take $x=0$ in 21, and use $u_{\lambda}$ to denote $u_{0, \lambda}$. By the $C^{1}$ regularity of $u$, there exists $r_{0}>0$ such that

$$
\frac{d}{d r}\left(r^{(n-2) / 2} u(r, \theta)\right)>0, \quad \forall 0<r<r_{0}, \theta \in S^{n-1},
$$

from which we deduce

$$
u_{\lambda}(y)<u(y), \quad \forall 0<\lambda<|y|<r_{0} .
$$

Because of (9), there exists some constant $\alpha>0$ such that

$$
u(y) \geq \frac{\alpha}{|y|^{n-2}}, \quad \forall|y| \geq r_{0} .
$$

Let $\lambda_{0}=\min \left\{\alpha^{1 /(n-2)}\left(\max \frac{}{B_{r_{0}^{+}}^{+}} u\right)^{1 /(2-n)}, r_{0}\right\}$. Then

$$
u_{\lambda}(y) \leq\left(\frac{\lambda_{0}}{|y|}\right)^{n-2} \max _{\overline{r_{0}}}^{+} u \leq \frac{\alpha}{|y|^{n-2}} \leq u(y), \quad \forall 0<\lambda<\lambda_{0},|y| \geq r_{0} .
$$

Now 21] with $x=0$ follows from 221 and the above. Lemma2 2 is established.

For $x \in \partial \mathbb{R}_{+}^{n}$, let

$$
\bar{\lambda}(x):=\sup \left\{\mu>0 \mid u_{x, \lambda} \leq u \text { on } \overline{\mathbb{R}_{+}^{n}} \backslash B_{\lambda}(x), \quad \forall 0<\lambda<\mu\right\} .
$$

Clearly, $\bar{\lambda}(x)>0$. On the other hand, $\bar{\lambda}(x)<\infty$ because of 9 .

Lemma 3. Let $u$ be as in Theorem 1 and assume that $u$ satisfies (19) and 20). Then, for all $x \in \partial \mathbb{R}_{+}^{n}$,

$$
u_{x, \bar{\lambda}(x)} \equiv u \quad \text { on } \mathbb{R}_{+}^{n} \backslash\{x\} .
$$

Proof. Without loss of generality, take $x=0$. We use the notation $\bar{\lambda}=\bar{\lambda}(0)$ and $u_{\lambda}=$ $u_{0, \lambda}$. By the definition of $\bar{\lambda}$,

$$
u_{\bar{\lambda}} \leq u \quad \text { on } \overline{\mathbb{R}_{+}^{n}} \backslash B_{\bar{\lambda}} .
$$

From now on, we always assume that (24) does not hold for $x=0$, and we will reach a contradiction. We first show that

$$
u-u_{\bar{\lambda}}>0 \quad \text { on } \overline{\mathbb{R}_{+}^{n}} \backslash \overline{B_{\bar{\lambda}}^{+}} .
$$

Indeed, if $\left(u-u_{\bar{\lambda}}\right)(\bar{x})=0$ for some $\bar{x} \in \mathbb{R}_{+}^{n} \backslash \overline{B_{\bar{\lambda}}^{+}}$, then using $\sqrt[83]{ }$ and hypotheses 44 and (6), we have

$$
F\left(A^{u \bar{\lambda}}\right)=1 \quad \text { on } \mathbb{R}_{+}^{n} \backslash \overline{B_{\bar{\lambda}}} .
$$


A calculation using $(8)$ yields

$$
\frac{\partial u_{\bar{\lambda}}}{\partial x_{n}}=c u_{\bar{\lambda}}^{n /(n-2)} \quad \text { on } \partial \mathbb{R}_{+}^{n} \backslash B_{\bar{\lambda}} .
$$

Arguing as in the proof of Lemma 2.1 in [12] (using hypotheses (5) and (77), we have, near $\bar{x}$,

$$
0=F\left(A^{u}\right)-F\left(A^{u \bar{\lambda}}\right)=L\left(u-u_{\bar{\lambda}}\right),
$$

where $L=-a_{i j}(x) \partial_{i j}+b_{i}(x) \partial_{i}+c(x)$ is an elliptic operator with continuous coefficients. By the strong maximum principle, $u-u_{\bar{\lambda}} \equiv 0$ near $\bar{x}$. This implies 24] for $x=0$, a contradiction.

If $\left(u-u_{\bar{\lambda}}\right)(\bar{x})=0$ for some $\bar{x} \in \partial \mathbb{R}_{+}^{n} \backslash \overline{B_{\bar{\lambda}}^{+}}$, we have

$$
\frac{\partial\left(u-u_{\bar{\lambda}}\right)}{\partial x_{n}}(\bar{x})=\left(c u^{n /(n-2)}-c u_{\bar{\lambda}}^{n /(n-2)}\right)(\bar{x})=0 .
$$

Since we still have (27) near $\bar{x}$, we apply the Hopf lemma to deduce that $u-u_{\bar{\lambda}} \equiv 0$ near $\bar{x}$, again leading to 24] for $x=0$, a contradiction. We have thus established (26).

Next we show that

$$
\lim _{y \in \mathbb{R}_{+}^{n},|y| \rightarrow \infty}|y|^{n-2}\left(u(y)-u_{\bar{\lambda}}(y)\right)>0 .
$$

Letting $x=y /|y|^{2}$, we have

$$
|y|^{n-2} u(y)=u_{0,1}(x), \quad|y|^{n-2} u_{\bar{\lambda}}(y)=\bar{\lambda}^{n-2} u\left(\frac{\bar{\lambda}^{2} y}{|y|^{2}}\right)=\bar{\lambda}^{n-2} u\left(\bar{\lambda}^{2} x\right)=: v(x) .
$$

By 19, , 20, and the conformal invariance of $(8)$, both $u_{0,1}$ and $v$ are $C^{2}$ solutions of 8 . We also know, from 26, that $u_{0,1}-v>0$ in $B_{1 / \bar{\lambda}}^{+}$. By the same arguments used in proving $u-u_{\bar{\lambda}}>0$ on $\partial \mathbb{R}_{+}^{n} \backslash \overline{B_{\bar{\lambda}}^{+}}$, we have $\left(u_{0,1}-v\right)(0)>0$, which implies 28 .

Since $u-u_{\bar{\lambda}}=0$ on $\partial B_{\bar{\lambda}} \cap \mathbb{R}_{+}^{n}$ and 26 ) holds, we can apply the Hopf lemma as in the proof of Lemma 2.1 in [12] (see also the outlines near [27) ) to obtain

$$
\frac{\partial\left(u-u_{\bar{\lambda}}\right)}{\partial v}>0 \quad \text { on } \partial B_{\bar{\lambda}} \cap \mathbb{R}_{+}^{n},
$$

where $v$ denotes the unit outer normal to $\partial B_{\bar{\lambda}}$.

Finally, we prove that

$$
\frac{\partial\left(u-u_{\bar{\lambda}}\right)}{\partial v}>0 \quad \text { on } \partial B_{\bar{\lambda}} \cap \partial \mathbb{R}_{+}^{n},
$$

where $v$ still denotes the unit outer normal to $\partial B_{\bar{\lambda}}$.

Let $\bar{x} \in \partial B_{\bar{\lambda}} \cap \partial \mathbb{R}_{+}^{n}$. Then as in the proof of Lemma 2.1 in [12], we have [27] near $\bar{x}$ with continuous coefficients. Clearly, for some constant $A>0$,

$$
\left|\frac{\partial\left(u-u_{\bar{\lambda}}\right)}{\partial x_{n}}\right|=\left|c\left(u^{n /(n-2)}-u_{\bar{\lambda}}^{n /(n-2)}\right)\right| \leq A\left(u-u_{\bar{\lambda}}\right) \quad \text { in }\left(\mathbb{R}_{+}^{n} \backslash \overline{B_{\bar{\lambda}}^{+}}\right) \cap B_{1}(\bar{x}) .
$$


By [27], and for a possibly larger $A$, we have

$$
a_{i j} \partial_{i j}\left(u-u_{\bar{\lambda}}\right)+b_{i} \partial_{i}\left(u-u_{\bar{\lambda}}\right) \leq A\left(u-u_{\bar{\lambda}}\right) \quad \text { in }\left(\mathbb{R}_{+}^{n} \backslash \overline{B_{\bar{\lambda}}^{+}}\right) \cap B_{1}(\bar{x}) .
$$

Now an application of Lemma 10.1 of [16] (with $\Omega=\left(\mathbb{R}_{+}^{n} \backslash \overline{B_{\bar{\lambda}}}\right) \cap B_{1}(\bar{x}), \sigma=x_{n}$, $\rho=|x|^{2}-\bar{\lambda}^{2}$, and our $u-u_{\bar{\lambda}}$ being the $u$ there) yields

$$
\frac{\partial\left(u-u_{\bar{\lambda}}\right)}{\partial v}(\bar{x})>0 .
$$

So we have established 30 .

Given 26, 28, 29], 30, and the positivity and continuity of $u$ on $\overline{\mathbb{R}_{+}^{n}}$, we can easily prove that there exists some $\epsilon>0$ such that

$$
u_{\lambda} \leq u \quad \text { on } \overline{\mathbb{R}_{+}^{n}} \backslash B_{\lambda}^{+}, \quad \forall \bar{\lambda} \leq \lambda \leq \bar{\lambda}+\epsilon,
$$

which violates the definition of $\bar{\lambda}$. Lemma 3 is established.

Proof of Theorem 1 under the additional hypotheses (19) and (20). Let $u$ be as in Theorem 1 and suppose $u$ satisfies (19) and 20]. By Lemma 3 and a calculus lemma used in [17] (see, e.g., Lemma 11.1 in [16]),

$$
u\left(x^{\prime}, 0\right) \equiv \frac{\hat{a}}{\left(\left|x^{\prime}-\bar{x}^{\prime}\right|^{2}+d^{2}\right)^{(n-2) / 2}} \quad \text { on } \mathbb{R}^{n-1},
$$

where $\bar{x}^{\prime} \in \mathbb{R}^{n-1}$, and $\hat{a}$ and $d$ are positive constants. Let $P=\left(\bar{x}^{\prime},-d\right)$ and define

$$
v(z):=\left(\frac{2 d}{|z-P|}\right)^{n-2} u\left(P+\frac{4 d^{2}(z-P)}{|z-P|^{2}}\right) .
$$

By the arguments in [17] and [2], as in the proof of Lemma 4.5 in [16], we know that $v$ is radially symmetric with respect to $Q:=\left(\bar{x}^{\prime}, d\right)$ in $B_{2 d}(Q)$. By the conformal invariance of the equation satisfied by $u$, we have

$$
F\left(A^{v}\right)=1, \quad A^{v} \in U, \quad v>0 \quad \text { in } \overline{B_{2 d}(Q)} .
$$

By Theorem 3 ,

$$
v(z) \equiv\left(\frac{\bar{a}}{1+\bar{b}|z-Q|^{2}}\right)^{(n-2) / 2} \text { in } \overline{B_{2 d}(Q)},
$$

where $\bar{a}>0$ and $1+\bar{b}(2 d)^{2}>0$. Comparing this with 31 , we must have $\bar{b}>0$. This, together with 31 , implies

$$
u(x) \equiv\left(\frac{a}{1+b|x-\bar{x}|^{2}}\right)^{(n-2) / 2} \quad \text { on } \mathbb{R}_{+}^{n},
$$

where $a=d^{-2} \hat{a}^{2 /(n-2)}, b=d^{-2}, \bar{x}=\left(\bar{x}^{\prime}, \bar{x}_{n}\right), \hat{a}, d, \bar{x}^{\prime}$ are given in 31, and $\bar{x}_{n}$ is some real number. 
Since $A^{u}(0)=2 a^{-2} b I$, we have $2 a^{-2} b I \in U$ and $F\left(2 a^{-2} b I\right)=F\left(A^{u}(0)\right)=1$. By the boundary condition for $u$ at $x=0$, we have $(n-2) a^{-1} b \bar{x}_{n}=c$. Theorem 1 is thus established under the additional hypotheses.

Proof of Theorem 1. By Lemma 2, there exists $\lambda_{0}>0$ such that

$$
u_{\lambda} \leq u \quad \text { on } \overline{\mathbb{R}_{+}^{n}} \backslash B_{\lambda}, \quad \forall 0<\lambda<\lambda_{0},
$$

where $u_{\lambda}=u_{0, \lambda}$ and $B_{\lambda}=B_{\lambda}(0)$. Let $w=u_{0,1}$. As in the proof of Lemma 2 and in the proof of Lemma 2.1 of [12], there exists some $\lambda_{1}>0$ such that

$$
w_{\lambda} \leq w \quad \text { on } \overline{\mathbb{R}_{+}^{n}} \backslash B_{\lambda}, \quad \forall 0<\lambda<\lambda_{1} .
$$

Rewrite (32) and (33) as

$$
\begin{array}{lll}
w_{\lambda} \leq w & \text { in } B_{\lambda}^{+}, & \forall \lambda>1 / \lambda_{0}, \\
w_{\lambda} \geq w & \text { in } B_{\lambda}^{+}, & \forall 0<\lambda<\lambda_{1},
\end{array}
$$

and let

$$
\begin{aligned}
& \underline{\lambda}:=\sup \left\{\mu\left|w_{\lambda}(x) \geq w(x), \forall 0<\right| x \mid \leq \lambda \leq \mu\right\}, \\
& \bar{\lambda}:=\inf \left\{\mu\left|w_{\lambda}(x) \leq w(x), \forall \lambda \geq \mu, 0<\right| x \mid \leq \lambda\right\} .
\end{aligned}
$$

If $\bar{\lambda} \leq \underline{\lambda}$, then $w_{\underline{\lambda}} \equiv w_{\bar{\lambda}} \equiv w$, and $u$ satisfies $[19]$ and 20 . In this case Theorem 1 has already been established. In the following, we assume that $\bar{\lambda}>\underline{\lambda}$ and we will reach a contradiction.

Clearly, $w_{\lambda}(0)=\frac{1}{\lambda^{n-2}} u(0)$, so we have

$$
\frac{1}{\bar{\lambda}^{n-2}} u(0) \leq w(0) \leq \frac{1}{\underline{\lambda}^{n-2}} u(0) .
$$

Since $\bar{\lambda}>\underline{\lambda}$, there must be at least one strict inequality in the above. Without loss of generality, we assume that

$$
w_{\bar{\lambda}}(0)=\frac{1}{\bar{\lambda}^{n-2}} u(0)<w(0) .
$$

This guarantees that there is no touching of $w_{\lambda}$ and $w$ near 0 for $\lambda$ close to $\bar{\lambda}$. Therefore, by the moving sphere arguments used earlier, we have, for $\lambda$ close to $\bar{\lambda}, w_{\lambda} \leq w$ in $B_{\lambda}$. This violates the definition of $\bar{\lambda}$. Theorem 1 is established.

\subsection{Proof of Theorem 2}

Let

$$
\alpha:=\liminf _{x \in \mathbb{R}_{+}^{n},|x| \rightarrow \infty}|x|^{n-2} u(x) \in[0, \infty] .
$$

Lemma 4. We have $\alpha>0$.

Proof. We follow the arguments of the proof of Lemma 4.1 in [16]. Let

$$
O:=\left\{\left.y \in \mathbb{R}_{+}^{n}|u(y)<| y\right|^{2-n}\right\} .
$$


We only need to show

$$
\liminf _{x \in O,|x| \rightarrow \infty}|x|^{n-2} u(x)>0 .
$$

We know that

$$
\begin{aligned}
& \Delta u \leq 0 \quad \text { in } O \\
& \frac{\partial u}{\partial x_{n}}=c u^{n /(n-2)} \leq(|c|+1)|y|^{-2} u \quad \text { on } \partial O \cap \partial \mathbb{R}_{+}^{n} .
\end{aligned}
$$

For $A>1$, let

$$
\xi(y):=\left|y-A e_{n}\right|^{2-n}+|y|^{1-n} .
$$

For large $A$ and $R=A^{2}$, we have

$$
\begin{cases}-\Delta \xi \leq 0 & \text { on } \mathbb{R}_{+}^{n} \backslash B_{R} \\ \frac{\partial \xi}{\partial x_{n}}(y) \geq \frac{|c|+1}{|y|^{2}} \xi(y), & \forall y \in \partial \mathbb{R}_{+}^{n} \backslash B_{R}\end{cases}
$$

Let $\bar{\epsilon}(A)>0$ be a small constant such that

$$
w:=u-\bar{\epsilon} \xi \geq 0 \quad \text { on } \partial\left(O \backslash B_{R}\right) \cap \mathbb{R}_{+}^{n} .
$$

It follows that

$$
\begin{cases}\Delta w \leq 0 & \text { on } O \backslash B_{R} \\ \frac{\partial w}{\partial x_{n}}(y)-\frac{|c|+1}{|y|^{2}} w(y) \leq 0, & \forall y \in \partial\left(O \backslash B_{R}\right) \cap \partial \mathbb{R}_{+}^{n}\end{cases}
$$

Clearly, $\lim \inf _{x \in O \backslash B_{R},|x| \rightarrow \infty} w(x) \geq 0$. By the maximum principle, $w \geq 0$ on $O \backslash B_{R}$. Hence

$$
\liminf _{x \in O,|x| \rightarrow \infty}|x|^{n-2} u(x) \geq \bar{\epsilon}>0 .
$$

Lemma 4 is proved.

Lemma 5. For any $x \in \partial \mathbb{R}_{+}^{n}$, there exists $\lambda_{0}(x)>0$ such that

$$
u_{x, \lambda} \leq u \quad \text { on } \mathbb{R}_{+}^{n} \backslash B_{\lambda}(x), \quad \forall 0<\lambda<\lambda_{0}(x) .
$$

Proof. Since we know that $\alpha>0$, Lemma 5 follows from the proof of Lemma 2

For $x \in \partial \mathbb{R}_{+}^{n}$, let $\bar{\lambda}(x)$ be defined as in 23. By Lemma $5 \bar{\lambda}(x)>0$.

Lemma 6. If $\alpha=\infty$, then

$$
\bar{\lambda}(x)=\infty, \quad \forall x \in \partial \mathbb{R}_{+}^{n} .
$$

If $\alpha<\infty$, then

$$
\bar{\lambda}(x)^{n-2} u(x)=\alpha, \quad \forall x \in \partial \mathbb{R}_{+}^{n} .
$$


Proof. By the definition of $\bar{\lambda}(x)$,

$$
u_{x, \lambda}(y) \leq u(y), \quad \forall 0<\lambda<\bar{\lambda}(x), \forall y \in \mathbb{R}_{+}^{n} \backslash B_{\lambda}(x) .
$$

It follows that

$$
\begin{aligned}
\lambda^{n-2} u(x) & =\liminf _{y \in \mathbb{R}_{+}^{n},|y| \rightarrow \infty}|y|^{n-2} u_{x, \lambda}(y) \\
& \leq \liminf _{y \in \mathbb{R}_{+}^{n},|y| \rightarrow \infty}|y|^{n-2} u(y)=\alpha, \quad \forall 0<\lambda<\bar{\lambda}(x) .
\end{aligned}
$$

If $\alpha<\infty$, we have

$$
\bar{\lambda}(x)^{n-2} u(x) \leq \alpha<\infty, \quad \forall x \in \partial \mathbb{R}_{+}^{n} .
$$

In fact we must have

$$
\bar{\lambda}(x)^{n-2} u(x)=\alpha .
$$

Indeed, if $\bar{\lambda}(x)^{n-2} u(x)<\alpha$, then

$$
\lim _{y \in \mathbb{R}_{+}^{n},|y| \rightarrow \infty}|y|^{n-2}\left(u(y)-u_{x, \bar{\lambda}(x)}(y)\right)=\alpha-\bar{\lambda}(x)^{n-2} u(x)>0,
$$

and the arguments in the proof of Lemma 3 show that the moving sphere procedure should not stop at $\bar{\lambda}(x)$, violating the definition of $\bar{\lambda}(x)$.

Now assume $\alpha=\infty$. Without loss of generality, we show $\bar{\lambda}:=\bar{\lambda}(0)=\infty$. We prove it by contradiction. Suppose $\bar{\lambda}<\infty$. By the definition of $\bar{\lambda}, 25$ holds. Since $\alpha=\infty$, we have

$$
\liminf _{y \in \overline{\mathbb{R}}_{+}^{n},|y| \rightarrow \infty}\left(u(y)-u_{\bar{\lambda}}(y)\right)|y|^{n-2}=\infty .
$$

This plays the same role as $(28)$ in the proof of Lemma 3 and the arguments there lead to a contradiction to the definition of $\bar{\lambda}$. Lemma 6 is established.

To prove Theorem 2, we first consider the case $\alpha<\infty$. Our proof goes along the lines of the proof of Theorem 1.3 in [14]. Our next lemma, whose proof is given towards the end of this section, is an analogue of Lemma 4.1 in [14].

Lemma 7. For $n \geq 3, a, d>0, c \in \mathbb{R}, p, q \in \mathbb{R}^{n-1}$ and $p \neq q$, let $\left.u \in C^{1} \overline{\left(B_{d}^{+}\right.} \backslash\{0\}\right)$ satisfy

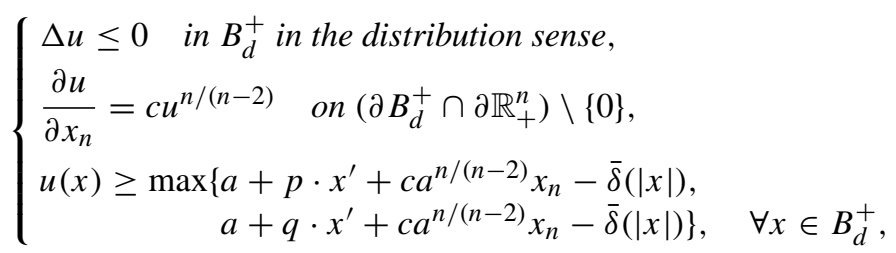

where $x^{\prime}=\left(x_{1}, \ldots, x_{n-1}\right), \bar{\delta}(r)>0$ and $\lim _{r \rightarrow 0^{+}} \bar{\delta}(r) / r=0$. Then

$$
\liminf _{x \in B_{1}^{+}, x \rightarrow 0} u(x)>a .
$$


Lemma 8. Under the hypothesis of Theorem 2 if $\alpha<\infty$, then $u$ is of the form 10 with $\bar{x}, a$ and $b$ given below $[10]$.

Proof. For $x \in \partial \mathbb{R}_{+}^{n}$, let

$$
\phi^{(x)}(y):=x+\frac{\bar{\lambda}(x)^{2}(y-x)}{|y-x|^{2}}, \quad \psi(y):=\frac{y}{|y|^{2}}, \quad w^{(x)}(y):=\left(u_{\phi^{(x)}}\right)_{\psi}=u_{\phi^{(x)} \circ \psi} .
$$

By the definition of $\bar{\lambda}(x)$,

$$
u \geq u_{\phi^{(x)}} \quad \text { on } \mathbb{R}_{+}^{n} \backslash B_{\bar{\lambda}(x)}(x), \quad \forall x \in \partial \mathbb{R}_{+}^{n} .
$$

By (34),

$$
w^{(x)}(0):=\bar{\lambda}(x)^{n-2} u(x)=\alpha, \quad \forall x \in \partial \mathbb{R}_{+}^{n} .
$$

We have

$$
\left\{\begin{array}{l}
u_{\psi} \in C^{2}\left(\mathbb{R}_{+}^{n}\right), \\
\Delta u_{\psi} \leq 0 \text { in } \mathbb{R}_{+}^{n} \text { since } \Delta u \leq 0 \text { in } \mathbb{R}_{+}^{n}, \\
\liminf _{\mathbb{R}_{+}^{n} \ni y \rightarrow 0} u_{\psi}(y)=\liminf _{z \in \mathbb{R}_{+}^{n},|z| \rightarrow \infty}|z|^{n-2} u(z)=\alpha,
\end{array}\right.
$$

and it is clear, for some $\delta(x)>0$ and by $(36)$, that

$$
\begin{aligned}
\left.w^{(x)} \in C^{2} \overline{\left(B_{\delta(x)}^{+}\right.}\right), & \forall x \in \partial \mathbb{R}_{+}^{n}, \\
u_{\psi} \geq w^{(x)}, & \text { in } B_{\delta(x)}^{+} .
\end{aligned}
$$

By (8) and the conformal invariance of the boundary condition satisfied by $u$,

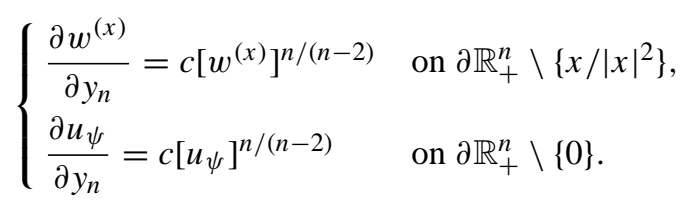

By Lemma7

$$
\nabla_{y^{\prime}} w^{(x)}(0)=\nabla_{y^{\prime}} w^{(0)}(0), \quad \forall x \in \partial \mathbb{R}_{+}^{n}
$$

So for $x=\left(x^{\prime}, 0\right)$,

$$
\begin{aligned}
\vec{V} & :=\nabla_{y^{\prime}} w^{(0)}(0)=(n-2) \bar{\lambda}(x)^{n-2} u(x) x+\bar{\lambda}(x)^{n} \nabla_{x^{\prime}} u(x)+\bar{\lambda}(x)^{n} \nabla_{x^{\prime}} u(x) \\
& =(n-2) \alpha x^{\prime}+\alpha^{n /(n-2)} u(x)^{n /(2-n)} \nabla_{x^{\prime}} u(x) .
\end{aligned}
$$

Thus we have

$$
\nabla_{x^{\prime}}\left[\frac{n-2}{2} \alpha^{n /(n-2)} u\left(x^{\prime}, 0\right)^{-2 /(n-2)}-\frac{n-2}{2}\left|x^{\prime}\right|^{2}+\vec{V} \cdot x^{\prime}\right]=0,
$$

which implies, for some $\bar{x}^{\prime} \in \mathbb{R}^{n-1}$ and $d \in \mathbb{R}$, that

$$
u\left(x^{\prime}, 0\right)^{-2 /(n-2)} \equiv \alpha^{-2 /(n-2)}\left|x^{\prime}-\bar{x}^{\prime}\right|^{2}+d \alpha^{-2 /(n-2)} .
$$


Since $u>0$, we have $d>0$ and

$$
u\left(x^{\prime}, 0\right) \equiv\left(\frac{\alpha^{2 /(n-2)}}{d+\left|x^{\prime}-\bar{x}^{\prime}\right|^{2}}\right)^{(n-2) / 2} .
$$

For simplicity, we take $\bar{x}^{\prime}=0$. By 34 and the above,

$$
\alpha=\bar{\lambda}(0)^{n-2} u(0)=\bar{\lambda}(0)^{n-2} \frac{\alpha}{d^{(n-2) / 2}},
$$

which gives $\bar{\lambda}:=\bar{\lambda}(0)=\sqrt{d}$. Since

$$
u_{\bar{\lambda}}(y)=\left(\frac{\bar{\lambda}}{|y|}\right)^{n-2} u\left(\frac{\bar{\lambda}^{2} y}{|y|^{2}}\right)
$$

we have, by 37,

$$
u_{\bar{\lambda}}\left(x^{\prime}, 0\right)=\frac{\bar{\lambda}^{n-2} \alpha}{\left(d\left|x^{\prime}\right|^{2}+\bar{\lambda}^{4}\right)^{(n-2) / 2}}=\frac{\alpha}{\left(\left|x^{\prime}\right|^{2}+d\right)^{(n-2) / 2}}=u\left(x^{\prime}, 0\right), \quad \forall x^{\prime} \in \mathbb{R}^{n-1} .
$$

Thus by the conformal invariance of the equation and the boundary condition satisfied by $u$, we have

$$
\begin{cases}F\left(A^{u}\right)=F\left(A^{u \bar{\lambda}}\right)=1, A^{u} \in U, A^{u_{\bar{\lambda}} \in U} & \text { in } \overline{\mathbb{R}_{+}^{n}} \backslash\{0\}, \\ u-u_{\bar{\lambda}}=0 & \text { on } \partial \mathbb{R}_{+}^{n} \backslash\{0\}, \\ \frac{\partial\left(u-u_{\bar{\lambda}}\right)}{\partial x_{n}}=c u^{n /(n-2)}-c u_{\bar{\lambda}}^{n /(n-2)}=0 & \text { on } \partial \mathbb{R}_{+}^{n} \backslash\{0\}, \\ u-u_{\bar{\lambda}} \geq 0 & \text { on } \mathbb{R}_{+}^{n} \backslash B_{\bar{\lambda}} .\end{cases}
$$

As usual, $u-u_{\bar{\lambda}}$ satisfies a linear second order elliptic equation and therefore, by the Hopf lemma and the strong maximum principle,

$$
u-u_{\bar{\lambda}} \equiv 0 \quad \text { on } \mathbb{R}_{+}^{n} .
$$

In particular, $u$ satisfies (19) and 20). So $u$ is of the form (10) by our earlier discussion of Theorem 2 under (19) and 20). Lemma 8 is established.

Lemma 9. Under the hypotheses of Theorem 2 except (11), if $\alpha=\infty$, then

$$
u\left(x^{\prime}, x_{n}\right) \equiv u\left(0^{\prime}, x_{n}\right), \quad \forall x^{\prime} \in \mathbb{R}^{n-1}, \forall x_{n} \geq 0 .
$$

Moreover $c \geq 0$, and if $c=0$, then $u$ must be a constant.

Proof. Since $\alpha=\infty$, we have, by Lemma 6 .

$$
\bar{\lambda}(x)=\infty, \quad \forall x \in \partial \mathbb{R}_{+}^{n} .
$$


i.e.,

$$
u_{x, \lambda} \leq u \quad \text { on } \mathbb{R}_{+}^{n} \backslash B_{\lambda}(x), \quad \forall 0<\lambda<\infty,
$$

which, by a calculus lemma (see, e.g., Lemma 11.3 in [16]), implies [38]. Let

$$
h(t):=u\left(0^{\prime}, t\right) \quad \text { for } t \geq 0 .
$$

Since $\Delta u \leq 0$, we have

$$
h^{\prime \prime}(t) \leq 0, \quad \forall t \geq 0
$$

so

$$
h^{\prime}(t) \leq h^{\prime}(s), \quad \forall t \geq s \geq 0 .
$$

Hence

$$
h(t)-h(s) \leq h^{\prime}(s)(t-s), \quad \forall t \geq s \geq 0,
$$

and

$$
h^{\prime}(s) \geq \liminf _{t \rightarrow \infty} \frac{h(t)-h(s)}{t-s} \geq 0, \quad \forall s \geq 0 .
$$

Since $\partial u / \partial x_{n}=c u^{n /(n-2)}$ on $\partial \mathbb{R}_{+}^{n}$,

$$
h^{\prime}(0)=\operatorname{ch}(0)^{n /(n-2)} .
$$

Since $h(0)>0$ and $h^{\prime}(0) \geq 0$, we have $c \geq 0$. If $c=0$, we have $h^{\prime}(0)=0$. Recall that $h^{\prime \prime}(t) \leq 0$, so

$$
h^{\prime}(t) \leq h^{\prime}(0)=0, \quad \forall t \geq 0 .
$$

On the other hand, $h^{\prime}(t) \geq 0$, so $h^{\prime}(t) \equiv 0$ and $h(t) \equiv h(0)$. Lemma 9 is established.

Proof of Theorem 2 If $\alpha<\infty$, the theorem follows from Lemma 8 If $\alpha=\infty$, then by Lemma 9. (38) holds, and we only need to rule out the possibility of $c>0$. For this aim, we make use of (11). As before, let

$$
h(t):=u\left(0^{\prime}, t\right), \quad \forall t \geq 0 .
$$

Claim. $\forall a>0$,

$$
\lim _{t \rightarrow \infty} \frac{h^{\prime}(t)}{h(t)^{a}}=0 .
$$

Indeed, if $\lim _{t \rightarrow \infty} h(t)=\infty$, then (39) is obvious, since $0 \leq h^{\prime}(t) \leq h^{\prime}(0)$. Otherwise, there exists some $b \in[h(0), \infty)$ such that $\lim _{t \rightarrow \infty} h(t)=b$. We also know that $\lim _{t \rightarrow \infty} h^{\prime}(t)$ exists since $h^{\prime \prime}(t) \leq 0$. So, by the boundedness of $h(t)$, we must have $\lim _{t \rightarrow \infty} h^{\prime}(t)=0$, which yields 39 .

Let $\left(\lambda_{1}, \ldots, \lambda_{n}\right)$ denote the eigenvalues of $A^{u}$. Then

$$
\left\{\begin{array}{l}
\lambda_{1}(t)=\cdots=\lambda_{n-1}(t)=-\frac{2}{(n-2)^{2}} \frac{h^{\prime}(t)^{2}}{h(t)^{2 n /(n-2)}}, \\
\lambda_{n}(t)=-\frac{2}{n-2} \frac{h^{\prime \prime}(t)}{h(t)^{(n+2) /(n-2)}}+\frac{2(n-1)}{(n-2)^{2}} \frac{h^{\prime}(t)}{h(t)^{2 n /(n-2)}} .
\end{array}\right.
$$


By (39) and the equation satisfied by $u$,

$$
\left\{\begin{array}{l}
f\left(\lambda_{1}, \ldots, \lambda_{n}\right)=1, \\
\lambda_{1}=o(1), \ldots, \lambda_{n-1}=o(1) \\
\lambda_{n}=-\frac{2}{n-2} h^{-(n+2) /(n-2)} h^{\prime \prime}+o(1) .
\end{array}\right.
$$

By assumption (11), there exists some $\delta>0$ such that

$$
\left|\left(\lambda_{1}, \ldots, \lambda_{n}\right)\right| \geq \delta
$$

so for large $t$,

$$
-\frac{2}{n-2} \frac{h^{\prime \prime}(t)}{h(t)^{(n+2) /(n-2)}} \geq \frac{\delta}{2},
$$

i.e.,

$$
-h^{\prime \prime}(t) \geq \frac{n-2}{4} \delta h(t)^{(n+2) /(n-2)}>\frac{n-2}{4} \delta h(0)^{(n+2) /(n-2)} .
$$

Integrating the above inequality twice leads to

$$
-h(t)+h(0)+h^{\prime}(0) t \geq \frac{n-2}{8} \delta h(0)^{(n+2) /(n-2)} t^{2}, \quad \forall t \geq 0 .
$$

Sending $t \rightarrow \infty$ in the above yields a contradiction to the positivity of $h$. Thus we have ruled out the possibility that $c>0$. Theorem 2 is established.

In the rest of this section, we prove Lemma 7 We use the notations

$e_{1}=(1,0, \ldots, 0), x=\left(x_{1}, \ldots, x_{n}\right)=\left(x^{\prime}, x_{n}\right), B_{r}^{+}=B_{r} \cap \mathbb{R}_{+}^{n}, \partial^{\prime} B_{1}^{+}:=\partial B_{1}^{+} \cap \mathbb{R}_{+}^{n}$.

Fixing some small $b>0$ to be specified later, let

$$
\phi_{b}(x)= \begin{cases}x_{1}, & \forall x \in \partial B_{1} \cap\left\{x \mid x_{1}>0, x_{n}>0\right\}, \\ 0, & \forall x \in \partial B_{1} \cap\left\{x \mid x_{1}<0, x_{n}>0\right\}, \\ -b, & \forall x \in \partial B_{1} \cap\left\{x \mid x_{n}<0\right\} .\end{cases}
$$

Define

$$
\phi(x):=\frac{1-|x|^{2}}{n \omega_{n}} \int_{\partial B_{1}} \frac{\phi_{b}(y)}{|x-y|^{n}} d S_{y}, \quad \forall x \in B_{1},
$$

where $\omega_{n}$ denotes the volume of the unit ball of $\mathbb{R}^{n}$. We know that $\phi \in C^{\infty}\left(B_{1}\right) \cap$ $C^{0}\left(\bar{B}_{1} \backslash \partial \mathbb{R}_{+}^{n}\right)$ and, after fixing some small $b>0$,

$$
\left\{\begin{array}{l}
\Delta \phi=0 \quad \text { in } B_{1}, \\
\phi(0)>0, \quad\|\phi\|_{L^{\infty}\left(B_{1}\right) \leq 1,} \\
\limsup _{B_{1} \ni x \rightarrow \bar{x}} \phi(x) \leq \max \left\{\bar{x}_{1}, 0\right\}, \quad \forall \bar{x} \in \partial B_{1} .
\end{array}\right.
$$


Claim. There exists a constant $\tilde{C}>0$, depending only on $n, b$, such that

$$
\frac{\partial \phi}{\partial x_{n}}(x) \geq \tilde{C}>0, \quad \forall x \in B_{1} \cap \partial \mathbb{R}_{+}^{n} .
$$

Indeed, consider

$$
\psi\left(x^{\prime}, x_{n}\right):=\phi\left(x^{\prime}, x_{n}\right)-\phi\left(x^{\prime},-x_{n}\right), \quad \eta(x):=\frac{b}{2} x_{n}, \quad \forall x=\left(x^{\prime}, x_{n}\right) \in B_{1}^{+} .
$$

We have

$$
\psi \geq \eta \quad \text { on } \partial^{\prime} B_{1}^{+} \cup\left(B_{1} \cap \partial \mathbb{R}_{+}^{n}\right)
$$

And for any $x \in B_{1}^{+}$,

$$
\begin{aligned}
\psi(x) & =\frac{1-|x|^{2}}{n \omega_{n}} \int_{\partial B_{1}} \frac{\phi_{b}(y)-\phi_{b}\left(y^{\prime},-y_{n}\right)}{|x-y|^{n}} d S_{y} \\
& =\int_{\partial^{\prime} B_{1}^{+}}\left(\phi_{b}(y)-\phi_{b}\left(y^{\prime},-y_{n}\right)\right)\left(\frac{1}{|x-y|^{n}}-\frac{1}{\left|x-\left(y^{\prime},-y_{n}\right)\right|^{n}}\right) d S_{y}>0,
\end{aligned}
$$

therefore

$$
\liminf _{x \rightarrow \tilde{x}}(\psi-\eta)(x) \geq 0, \quad \forall \tilde{x} \in \partial B_{1}^{+} .
$$

By the maximum principle, $\psi \geq \eta$ in $B_{1}^{+}$. Since $\psi-\eta=0$ on $B_{1} \cap \partial \mathbb{R}_{+}^{n}$, we have

$$
\frac{\partial \psi}{\partial x_{n}} \geq \frac{\partial \eta}{\partial x_{n}}=\frac{b}{2} \quad \text { on } B_{1} \cap \partial \mathbb{R}_{+}^{n} .
$$

The Claim is proved.

Proof of Lemma 7. We only need to prove the lemma with $a=1, p-q=e_{1}:=$ $(1, \ldots, 1)$. Indeed, replacing $u$ by $(1 / a) u, c$ by $c a^{2 /(n-2)}, p$ by $(1 / a) p$ and $q$ by $(1 / a) q$, we can assume $a=1$. After a rotation, we can assume $p-q=\lambda e_{1}$ for some $\lambda>0$. Replacing $u(x)$ by $u(x / \lambda), c$ by $c / \lambda, p, q$ by $p / \lambda, q / \lambda$ respectively, we can also assume $p-q=e_{1}$.

Since $\lim _{r \rightarrow 0} \bar{\delta}(r) / r=0$, there exists $0<\bar{r}<d$ such that

$$
\frac{\bar{\delta}(r)}{r} \leq \frac{1}{2} \phi(0), \quad \forall 0<r<\bar{r},
$$

where $\phi$ is defined by 40 . For $0<r<\bar{r}$, we consider, for $0<s<r$,

$$
\phi^{r}(x):=1+c x_{n}+q \cdot x^{\prime}+r \phi\left(\frac{x}{r}\right)-\frac{s^{n-2} d}{|x|^{n-2}}-\sup _{(0, r]} \bar{\delta}, \quad \forall x \in \overline{B_{r} \backslash B_{s}} .
$$

By the equations for $u$ and $\phi$, we have

$$
\Delta\left(u-\phi^{r}\right) \leq 0 \quad \text { in } B_{r}^{+} \backslash B_{s}^{+} .
$$


By the last lines in 35 and 41 ,

$$
\limsup _{B_{r}^{+} \ni x \rightarrow \bar{x}}\left(u(x)-\phi^{r}(x)\right) \geq 0, \quad \forall \bar{x} \in \partial B_{r} \cap \overline{\mathbb{R}_{+}^{n}} .
$$

Indeed, if $\bar{x}_{1} \geq 0$, we have, using $p-q=e_{1}$,

$$
u(x)-\phi^{r}(x) \geq\left(1+p \cdot x^{\prime}+c x_{n}-\bar{\delta}(|x|)\right)-\phi^{r}(x) \geq x_{1}-r \phi(x / r),
$$

from which we deduce (44). If $\bar{x}_{1}<0$, estimate (44) follows from

$$
u(x)-\phi^{r}(x) \geq\left(1+q \cdot x^{\prime}+c x_{n}-\bar{\delta}(|x|)\right)-\phi^{r}(x) \geq-r \phi(x / r) .
$$

Since $\|\phi\|_{L^{\infty}\left(B_{1}\right)} \leq 1$, we have

$$
\phi^{r}(x)<1+c x_{n}+q \cdot x^{\prime}-\sup _{(0, r]} \bar{\delta}, \quad \forall x \in \partial B_{s} \cap \overline{\mathbb{R}_{+}^{n}} .
$$

Thus, by the last line in 35 ,

$$
u-\phi^{r} \geq 0 \quad \text { on } \partial B_{s} \cap \overline{\mathbb{R}_{+}^{n}} .
$$

Claim. There exists $\tilde{r} \in(0, \bar{r}]$ such that for all $0<s<r<\tilde{r}$,

$$
\inf _{B_{r}^{+} \backslash B_{s}^{+}}\left(u-\phi^{r}\right) \geq 0 .
$$

Suppose not; we have, by (44), 45), and the strong maximum principle,

$$
\inf _{B_{r}^{+} \backslash B_{s}^{+}}\left(u-\phi^{r}\right)=\left(u-\phi^{r}\right)(\bar{x})<0 \quad \text { for some } \bar{x} \in\left(\partial \mathbb{R}_{+}^{n} \cap\left(B_{r} \backslash \bar{B}_{s}\right)\right) .
$$

At $\bar{x}$,

$$
0 \leq \frac{\partial\left(u-\phi^{r}\right)}{\partial x_{n}}=c u^{n /(n-2)}(\bar{x})-c-\frac{\partial \phi}{\partial x_{n}}\left(\frac{\bar{x}}{r}\right) \leq c u^{n /(n-2)}(\bar{x})-c-\tilde{C},
$$

where $\tilde{C}$ is the constant in 42 . By the last line in 35 , we have, for some universal positive constant $C$,

$$
u(\bar{x}) \geq 1-C|\bar{x}| \geq 1-C r .
$$

On the other hand,

$$
u(\bar{x}) \leq \phi^{r}(\bar{x}) \leq 1+C r .
$$

We deduce from 47, using the above two estimates,

$$
0 \leq C r-\tilde{C},
$$

which is impossible if we choose $\tilde{r}<\min \{\tilde{C} / C, \bar{r}\}$. 46 is established.

Sending $s \rightarrow 0$ in 46 , we obtain

$$
u(x) \geq 1+c x_{n}+q \cdot x^{\prime}+r \phi(x / r)-\sup _{(0, r]} \bar{\delta}, \quad \forall x \in B_{r}^{+} .
$$


Sending $x \rightarrow 0$, we have, by 43$]$,

$$
\liminf _{B_{1}^{+} \ni x \rightarrow 0} u(x) \geq 1+r \phi(0)-\sup _{(0, r]} \bar{\delta}>1 .
$$

Lemma77is established.

Acknowledgements. Part of this paper was completed while the second author was a visiting member at the Institute for Advanced Study in Fall 2003. He thanks J. Bourgain and IAS for providing him with the excellent environment, as well as for the financial support through NSF-DMS0111298. Part of the work of the second author is also supported by NSF-DMS-0100819.

\section{References}

[1] Ambrosetti, A., Malchiodi, A., Li, Y.Y.: On the Yamabe problem and the scalar curvature problems under boundary conditions. Math. Ann. 322, 667-699 (2002) Zbl 1005.53034 MR 1905107

[2] Bianchi, G.: Non-existence of positive solutions to semilinear elliptic equations on $\mathbb{R}^{n}$ or $\mathbb{R}_{+}^{n}$ through the method of moving planes. Comm. Partial Differential Equations 22, 1671-1690 (1997) Zbl 0910.35048 MR 1469586

[3] Brendle, S.: A family of curvature flows on surfaces with boundary. Math. Z. 241, 829-869 (2002) Zbl 1036.53044 MR 1942242

[4] Chang, S. Y. A., Gursky, M., Yang, P.: A conformally invariant sphere theorem in four dimensions. Publ. IHES 98, 105-143 (2003) Zbl 1066.53079 MR 2031200

[5] Cherrier, P.: Problèmes de Neumann non linéaires sur les variétés riemanniennes. J. Funct. Anal. 57, 154-206 (1984) Zbl 0552.58032 MR 0749522

[6] Escobar, J. F.: Uniqueness theorems on conformal deformation of metrics. Sobolev inequalities, and an eigenvalue estimate, Comm. Pure Appl. Math. 43, 857-883 (1990) Zbl 0713.53024 MR 1072395

[7] Escobar, J. F.: Conformal deformation of a Riemannian metric to a scalar flat metric with constant mean curvature. Ann. of Math. 136, 1-50 (1992) Zbl 0766.53033 MR 1173925

[8] Escobar, J. F.: The Yamabe problem on manifolds with boundary. J. Differential Geom. 35, 21-84 (1992) Zbl 0771.53017 MR 1152225

[9] Escobar, J. F.: Conformal deformation of a Riemannian metric to a constant scalar curvature metric with constant mean curvature on the boundary. Indiana Univ. Math. J. 45, 917-943 (1996) Zbl 0881.53037 MR 1444473

[10] Han, Z. C., Li, Y.Y.: The existence of conformal metrics with constant scalar curvature and constant boundary mean curvature. Comm. Anal. Geom. 8, 809-869 (2000) Zbl 0990.53033 MR 1792375

[11] Han, Z. C., Li, Y.Y.: The Yamabe problem on manifolds with boundary: Existence and compactness results. Duke Math. J. 99, 489-542 (1999) Zbl 0945.53023 MR 1712631

[12] Li, A., Li, Y.Y.: On some conformally invariant fully nonlinear equations. Comm. Pure Appl. Math. 56, 1416-1464 (2003) Zbl pre02002141 MR 1988895

[13] Li, A., Li, Y.Y.: On some conformally invariant fully nonlinear equations, Part II: Liouville, Harnack and Yamabe. arXiv:math.AP/0403442 (2004)

[14] Li, A., Li, Y.Y.: On some conformally invariant fully nonlinear equations, Part II: Liouville, Harnack and Yamabe. Acta Math., to appear 
[15] Li, A., Li, Y.Y.: On some conformally invariant fully nonlinear equations, Part III. In preparation

[16] Li, Y.Y., Zhang, L.: Liouville type theorems and Harnack type inequalities for semilinear elliptic equations. J. Anal. Math. 90, 27-87 (2003) MR 2001065

[17] Li, Y.Y., Zhu, M.: Uniqueness theorems through the method of moving spheres. Duke Math. J. 80, 383-417 (1995) Zbl 0846.35050 MR 1369398 